\title{
CALENDAR OF EVENTS
}

This selected listing of upcoming events is offered as a service to our readers. It provides brief information on various conferences and meetings which are of more than local significance, and covers the period of approximately one year beyond the publication date of each issue. We can of course assume no responsibility for the complete accuracy of the information on which individual entries are based. Supplementary entries and updated details are incorporated with each issue, insofar as these come in the meantime to our attention.

June $4-9,1983$

Special Libraries Association (SLA) 74th Annual Conference: "Removing Information Barriers". Location: New orleans, LA (USA). Contact: Conference and Exhibits Coordinator, SLA, 235 Park Avenue South, New York, NY 10003, USA. Tel.: (212) 477-9250.

June $6-8,1983$

Data Base '83. Location: Budapest, Hungary. Contact: Data Base ' 83 organizing Committee, c/o National Technical Information Centre and Library, Reviczky u.6, P.0.Box 12, 1428 Budapest, Hungary. Tel.: 336-309. Telex: omikk h 22-4944.

June $6-8,1983$

National Educational Computer Conference. Location: Baltimore, MD (USA). Contact: NECC, P.0.Box 639, Silver Spring, MD 20901, USA. Tel.: (301)589-3386.

June $8-10,1983$

Cinquième Congrès National sur 1'Information, la Documentation et le Transfert des Connaissances. Location: Grenoble, France. Contact: Secrétariat du Congrès, Alpes Congrès, Avenue d'Innsbruck, 38029 Grenoble Cedex, France.

June $16-21,1983$

Canadian Library Association Annual Conference. Location: Winnipeg, Manitoba, Canada. Contact: William F. Birdsall, University Librarian, Dalhousie University Library, Halifax, Nova Scotia B3H 448 , Canada.

0167-8329/83/\$03.00 (C) 1983 Elsevier Science Publishers B.V. (North-Holland) 
June 25 - July 1, 1983

American Library Association (ALA) Annual Conference. Location: Los Angeles, CA (USA). Contact: American Library Association, 50 East Huron Street, Chicago, IL 60611, USA. Tel.: (312) 944-6780.

June 28 - July 1, 1983

Institute of Information Scientists (IIS) Conference. Location: Oxford, UK. Contact: Christine Baker, Pilkington Library, Loughborough University of Technology, Loughborough, Leicestershire LE11 3TU, UK. Tel.: (0509) 63171, ext. 5047/8.

July $8-10,1983$

Society of Indexers (United Ringdom) 1983 Weekend Conference: "New Horizons for Indexing". Location: Bristol, UK. Contact: Moira Greenhalgh, 38 Manton Hollow, Manton, Marlborough, UK. Tel.: (0672) 53862 .

July 19-22, 1983

Third International Conference on User Education. Location: Edinburgh, UK. Contact: Ian Malley, British Library Information officer for User Education, Library, Loughborough University of Technology, Loughborough, Leicestershire LE11 3TU, UK, or ICUE, 13 Caernarvon Close, Shepshed, Leicestershire LE12 9QB, UK.

July 25-29, 1983

Informatics in Blementary Bducation. Location: Kiel, F.R.G. Contact: Mr. J.D. Tinsley, City of Birmingham Education, Council House, Margaret Street, Birmingham B3 3BU, UK.

August $10-15,1983$

Annual Conference of the International Association of School Librarianship. Location: Bad Segeberg, F.R.G. Contact: Anke Matthies, Conference Chairman, Stadtbüchereien Norderstedt, Europaallee 36, Norderstedt, F.R.G. Tel.: (040) 5231808.

August $12-14,1983$

First Annual Conference, Research Institute for Information Science and Engineering (RIISB): "Education for Information Professionals". Location: Washington, DC (USA). Contact: RIISE, P.O. BOX 7551, Pittsburgh, PA 15213, USA. Tel.: (412) 624-5205.

August $21-27,1983$

49th International Federation of Library Associations and Institutions (IFLA) General Conference: "Libraries in a Technological World". Location: Munich, F.R.G. Contact: IFLA, P.O.Box 95312, $2509 \mathrm{CH}$ The Hague, The Netherlands. Tel.: (070) 140884 . 
August 31 - September 7, 1983

International Association of Orientalist Librarians 1983

Conference. Location: Tokyo and Kyoto, Japan. Contact: IAOL Secretary-Treasurer, c/o Institute of Library Science, 3rd Floor, Gonzalez Hall, University of the Philippines, Diliman, Quezon City 3004, Philippines.

August 31 - September 9, 1983

Library Association (United Ringdom) 2nd International Summer Workshop: "The Developing Workforce". Location: London, UK. Contact: Short Courses Organiser, Library Association, 7 Ridgmount Street, London WC1E 7AE, UK. Tel.: (01) $636-7543$.

September $5-7,1983$

IRFIS 5: Fifth International Research Forum in Information Science. Location: Heidelberg, F.R.G. Contact: Dr. Hans Dietschmann, IRFIS 5 Coordinator, Gesellschaft für Information und Dokumentation, Lyonnerstrasse 44-48, 6000 Frankfurt a.M. 71, F.R.G.

September $13-16,1983$

ASLIB 56th Annual Conference: "Information for National Recovery: Needs - Resources - Technology". Location: Stirling, UK. Contact: Conference Organiser, ASLIB, 3 Belgrave Square, London SW1X 8PL, UK. Tel.: (01) 235-5050. Telex: 23667.

September $17-21,1983$

Library and Information Technology Association (LITA) National Conference: "Information and Technology: at the Crossroads". Location: Baltimore, MD (USA). Contact: LITA, American Library Association, 50 East Huron Street, Chicago, IL 60611, USA. Tel.: (312)944-6780.

September $18-20,1983$

Association of Information and Dissemination Centers

(ASIDIC) Fall Meeting. Location: Philadelphia, PA (USA) . Contact: ASIDIC, P.O. Box 8105, Athens, GA 30603, USA. Tel.: (404) 542-3106.

September 19-22, 1983

Library Association (United Kingdon) Annual

Conference: "Access to Published Information". Location:

Torquay, UK. Contact: Conferences officer, Library

Association, 7 Ridgmount Street, London WC1E 7AE, UK. Tel.: (01) $636 \rightarrow 7543$. 
September 26-29, 1983

6th National Special Libraries Conference: "Management of Resource Sharing in Special Libraries". Location: Perth, Australia. Contact: Chairman, Conference Committee, 6th National Special Libraries Conference, Mr. Bruce Bott, Law Library, Supreme Court, Barrack Street, Perth 6000, Western Australia. Tel.: (09) 325-9433.

September 27-29, 1983

Annual Meeting of FID/II: "Training of Information

Personnel". Location: Budapest, Hungary. Contact: J.R. Perez Alvarez-Ossorio, c/o IIDCT, Joaquín Costa 22, Madrid 6, spain. Tel.: (91) 261-4808.

October $2-6,1983$

American Society for Information Science (ASIS) 46th Annual Heeting: "Productivity in the Information Age". Location: Arlington, VA (USA). Contact: ASIS, 1010 Sixteenth Street NW, Washington, DC 20036. Tel.: (202)659-3644.

October 10-12, 1983

Online '83: "New Online Horizons - the Software Connection". Location: Chicago, IL (USA). Contact: Online Inc., 11 Tannery Lane, Weston, CT 06883, USA. Tel.:

(203) 227-8466.

October $10-13,1983$

Info ' 83: Information Management Exposition and Conference. Location: New York, NY (USA). Contact: Clapp \& Poliak Inc., 708 Third Avenue, New York, NY 10017, USA. Tel.: (212)661-8410.

October $16-23,1983$

Training Course in Accessibility and Dissemination of Non-bibliographic Data in Science and Technology. Location: Stockholm, Sweden. Contact: Dr. S. Schwarz, Director, Royal Institute of Technology Library, 10044 Stockholm, Sweden. (Participation limited).

October 19-20, 1983

Association of Research Libraries (ARL), USA, Membership Meeting. Location: Chapel Hill, NC (USA). Contact: ARL, 1527 New Hampshire Avenue NW, Washington, DC 20036, USA. Tel.: (202) 232-2466.

December $8-10,1983$

International Conference on New Technology in the Iibrary/Information School Curriculum. Location: London, UK. Contact: Ms. T.C. Bomford, British Library Research \& Development Department, 2 Sheraton street, London W1V 4BH, UK. 
January 7-12, 1984

American Library Association (ALA) Midwinter Conference. Location: Washington, DC (USA). Contact: American Library Association, 50 East Huron Street, Chicago, IL 60611, USA. Tel.: (312) 944-6780.

January 23-27, 1984 Special Libraries Association (SLA) winter meeting.

Location: Colorado Springs, CO (USA). Contact: Conference and Exhibits Coordinator, SLA, 235 Park Avenue South, New York, NY 10003, USA. Tel.: (212)477-9250.

May 25-31, 1984

Medical Library Association Annual Conference. Location: Denver, CO (USA). Contact: MLA, 919 North Michigan Avenue, Suite 3208, Chicago, IL 60611, USA. Tel.: (312) 266-2456. 